\title{
Spin current-induced by a sound wave
}

\author{
Igor. I. Lyapilin ${ }^{\text {a) }}$ \\ Institute of Metal Physics, Ural Division of Russian Academy of Sciences, S. Kovalevskaya Street 18, 620990 \\ Ekaterinburg, Russia
}

(Received 5 April 2012; revised 29 January 2013; accepted 12 February 2013)

\begin{abstract}
The interaction of conduction electrons with a longitudinal sound wave propagating in a crystal in a constant magnetic field is investigated. It is shown that the transverse spin current arises when the longitudinal sound wave propagation through the system. The average power absorbed by the spin subsystem of the conduction electrons and the spin-Hall conductivity have a resonant character. (C) 2013 Acoustical Society of America. [http://dx.doi.org/10.1121/1.4794380]
\end{abstract}

PACS number(s): 43.25.Ts, 43.35.Rw [JDM]

Pages: 1894-1896

\section{INTRODUCTION}

Electronic spin transport in low-dimensional and nanoscale systems is the subject of a novel and rapidly developing field of spintronics. An ultimate goal of this field is to create the possibility of coherent spin manipulation. Typically, the spin transport is highly dependent on coupling between spin and orbital degrees of freedom. In recent years, effects due to a response of electron spin degrees of freedom to an external perturbation acting on the kinetic (translational) degrees of freedom are of great interest to researchers. Combined electric dipole resonance when an interaction between the conduction electrons and an alternating electric field leads to the Zeeman resonance frequency can serve as a case in point of the effect mentioned above. This response also is exhibited as the Spin Hall Effect (SHE), ${ }^{1}$ which consists of the appearance of spin current in a direction perpendicular to the normal current when switching an electric field. SHE has been observed experimentally both at low and room temperatures. ${ }^{2}$ This effect leads to spin density accumulation on the lateral surfaces of a current-carrying specimen. In both cases, only the kinetic degrees of freedom of electrons are directly affected by the external perturbation (the electric field), which is transferred to the spin subsystem through the spin-orbital interaction.

The electronic motion can be driven not only by an electric field but also by a temperature gradient. In principle, besides electric and heat currents, a temperature gradient may also generate a spin current in a spintronic system. The background of the experiments and analysis the Spin Seebeck Effect in ferromagnetic metals are discussed in the papers. ${ }^{3}$ It is surprising that this effect is also observed in nonconducting crystals. The number of similar studies is rapidly growing so that it makes sense to speak of a new direction in spintronics, called caloritronica, as far as heat flux influence on the spin currents and vice versa is concerned. ${ }^{4}$

It is interesting to consider mechanisms of the interaction with external fields the energy of which is transmitted simultaneously to both subsystems (kinetic and spin). An interaction between conduction electrons and sound waves

\footnotetext{
a) Author to whom correspondence should be addressed. Also at: Ural Federation University, Mira Str. 19, 620002 Ekaterinburg, Russia. Electronic mail: Lyapilin@imp.uran.ru
}

can be given as an example. Note that the response in interacting between the spin subsystem (similar to SHE) of electrons and sound waves, having a resonant character, has been observed experimentally. ${ }^{5}$ The purpose of the present paper is to study behavior of the electronic system in the field of sound waves and also to analyze conditions of initiation of the response of the spin subsystem.

\section{INTERACTIONS}

There are several mechanisms responsible for the energy absorption by free electrons from ultrasonic waves: (1) Sound modulation of the spin-orbit coupling between conduction electrons and a crystal lattice; ${ }^{6}$ (2) sound modulation of the interaction between spin and kinetic degrees of freedom of conduction electrons in crystals without an inversion center as to electron $g$-factor, which depends on a momentum; ${ }^{7}$ (3) an interaction between electron spin and an alternating magnetic field accompanying a sound wave; ${ }^{8}$ (4) sound modulation of dipole-dipole interactions between electron spins. ${ }^{8}$ The preceding mechanisms differ from each other not only in interaction intensity but also in line width and in a position of resonance frequencies.

In general case, the interaction between the conduction electrons and the sound waves has a resonant character. The resonance arises either when frequency of sound $\omega$ coincides with spin precession frequency $\omega_{s}$ or when other frequencies are linear combinations of the Zeeman $\omega_{s}$ and cyclotron $\omega_{0}$ frequencies. In contrast to paramagnetic resonance, acoustic spin resonance (ASR) can be observed both in longitudinal and in transverse polarization of a sound wave. We have derived quantum kinetic equations to describe evolution of spin components and examined the effects associated with the absorption and redistribution of energy between the subsystems of the kinetic and spin degrees of freedom.

In case under consideration, all interactions between electrons and the sound wave

$$
\mathrm{u}(\mathrm{x}, t)=\sum_{q} \mathrm{u}(\mathrm{q}) e^{i q x+i \omega t}
$$

(where $u(q)$ is the amplitude of the sound wave with a wave vector $\mathbf{q}, \omega=s q, s$ is sound velocity) can be represented as a Hamiltonian of the following form: 


$$
H_{e f}(t)=\sum_{i n \mathbf{q}} \Phi_{-i}^{-n}(\mathbf{q}) u^{i}(\mathbf{q}) e^{i \omega t} T^{n}(\mathbf{q})
$$

where $T^{n}(\mathbf{q})$ is the tensor operator, which depends on the group indices $n=\left(\mu, \alpha_{1}, \alpha_{2}, \ldots\right)$.

The explicit form of the operator is defined by a particular crystal structure. $\Phi_{-i}^{-n}(\mathbf{q})$ is the C-numerical matrix. In other cases, the interactions with sound depend both on the translational and spin operators. Hence, the tensor operator has the form

$$
T^{n}(\mathbf{q})=\sum_{j}\left\{S_{j}^{\mu} P_{j}^{\alpha} ; e^{i q x_{j}}\right\} \equiv T^{\mu \alpha}(\mathbf{q}) .
$$

Here, $x_{j}, P_{j}^{\alpha}, S_{j}^{\mu}$ are the operators of coordinates, the momentum, and spin of the $j$ th electron, respectively. The indices $\mu$, $\alpha$ take the values $(0,+,-)$. The brackets $\{. ., \ldots\}$ denote the symmetrized product of operators. $A^{ \pm}=A_{x} \pm i A_{y}$.

Let us briefly discuss the structure of the interaction of sound and the spin degrees of freedom of conduction electrons. As to the preceding mechanisms, the structure is different, if the operator $T^{n}$ does not depend on the momentum operator, i.e., such a mechanism takes place in $\mathrm{Bi}$ and $\mathrm{Si}$, the mechanism (3) in CdS and mechanism (2) in general, and if, for the mechanism (1) in $\mathrm{Na}$ and $\mathrm{K}$ and for the mechanism (3) in Ge and InSb. ${ }^{9}$

The total Hamiltonian of the conduction electrons, interacting with the lattice displacement field $u(x, t)$ and scatterers in a constant magnetic field $\mathbf{H}=(0,0, H)$, is the sum of operators such as operators of the kinetic and the Zeeman electron energies, $H_{k}$, and $H_{s}$, respectively, an operator of the interaction between the electrons and scatterers $H_{e l}$ and the Hamiltonian of the lattice $H_{l}$. The interaction between the electrons and the sound wave is described by the Hamiltonian $H_{e f}(t)$. We have examined the effects associated with the absorption and redistribution of energy between the subsystems of the kinetic and spin degrees of freedom in the vicinity of the resonance frequencies in a quadratic approximation with respect to the displacement amplitudes.

\section{KINETIC EQUATIONS}

The problem we are interested in is evolution of the spin subsystem of conduction electrons. We assume that the sound wave propagates along $x$ and the operator $T^{n}(q)=T^{\alpha \gamma}(q)$. Using the explicit form of the operator $T^{+-}(q)$, we obtain macroscopic equations of motion for the transverse spin components. The macroscopic equations have been obtained by use of the nonequilibrium statistical operator $\rho(t)$ in a linear approximation with respect to sound wave-intensity: ${ }^{10}$

$$
\begin{aligned}
\rho(t, 0)= & \left.\rho_{q}(t, 0)-i \int_{-\infty}^{0} d t^{\prime} e^{\epsilon t^{\prime}} e^{i t^{\prime} L} L_{e f} \rho_{q}\left(t+t^{\prime}, 0\right)\right\}, \\
& \epsilon \rightarrow+0, \quad e^{i t L} A=e^{i t H / \hbar} A e^{-i t H / \hbar}, \\
& i L_{i} A=(i \hbar)^{-1}\left[A, H_{i}\right],
\end{aligned}
$$

where $\rho_{q}(t)=\exp \{-S(t, 0)\}$ is the quasi-equilibrium statistical operator. $S(t)$ is the entropy operator.
Now, the commutators $\dot{S}^{ \pm}=(i \hbar)^{-1}\left[S^{ \pm}, H\right]$ need to be calculated. Upon averaging and using (3), one arrives at

$$
\begin{aligned}
\left\langle\dot{S}^{ \pm}\right\rangle= & \pm \frac{2 i}{\hbar} \sum_{q} \Phi_{x}^{z-}(q) u^{x}(q) e^{i \omega t}\left\langle T^{z-}(q)\right\rangle \\
& +\left\langle\dot{S}_{(l)}^{ \pm}\right\rangle \mp \omega_{s}\left\langle S^{ \pm}\right\rangle .
\end{aligned}
$$

Here $\langle A\rangle=S p\{\rho(t) A\}, \dot{S}_{l}^{ \pm}=(i \hbar)^{-1}\left[S^{ \pm}, H_{e l}\right]$.

The first term on the right-hand side of the Eq. (4) determines power $Q_{s}$ absorbed by the spin degrees of freedom as a result of an interaction the latter with the field of sound waves. $\left\langle T^{z-}(q)\right\rangle=\left\langle S^{z} v^{-}+v^{-} S^{z}\right\rangle / 2=J^{s}$ is the spin current. Second term in Eq. (4) defines the spin relaxation processes.

$$
\begin{gathered}
Q_{s}=\beta \sum_{q} \omega^{2}\left|\Phi_{x}^{z-}(q) u^{x}(q)\right|^{2} \\
\times \operatorname{Re} \int_{-\infty}^{0} d t e^{t(\epsilon-i \omega)}\left(T^{z-}(q) ; T^{z+}(-q)\right), \\
\left(T^{z-}(q) ; T^{z+}(-q)\right)=\int_{0}^{1} d \tau\left\langleT ^ { z - } ( q ) \rho _ { 0 } ^ { \tau } \left( T^{z+}(-q)\right.\right. \\
\left.\left.-\left\langle T^{z+}(-q)\right\rangle_{0}\right) \rho_{0}^{-\tau}\right\rangle_{0} .
\end{gathered}
$$

$\beta$ is the reciprocal temperature, $\rho_{0}$ is the equilibrium statistical operator. $\langle A\rangle_{0}=S p A \rho_{0}$.

As follows from the expression for the power absorbed by the spin subsystem that the spin flow oriented along the $y$ axis arises when the sound wave propagates along the $x$ axis.

General conclusions concerning both the spin subsystem behavior and the possibility of observing the spin effect caused by the interaction between electrons and sound waves can be done by considering the correlation function (5).

The equation of motion for the operators defines a precession frequency of $T^{n}$ in the magnetic field (resonance frequencies) $\left[i L_{0} T^{z-}(q)\right]$ and a nonuniform distribution of the diffusion flow $\left[i L_{v} T^{z-}(q)\right]$. We introduce Green's functions

$$
G_{z+}^{z-}\left(t-t^{\prime}\right)=\theta\left(t-t^{\prime}\right) e^{\epsilon\left(t^{\prime}-t\right)}\left(T^{z-}(q, t), T^{z+}\left(-q, t^{\prime}\right)\right) .
$$

We can write a chain of equations for Green's functions where we retain only the terms up to second order in $H_{e l}$. We restrict ourselves to null approximation with respect to thermodynamic forces in the terms of the first and second orders in $H_{e l}$. Then we obtain the following expression for Green's function

$$
\begin{aligned}
& \operatorname{Re} G_{z+}^{z-}=\frac{\left(T^{z-}(q), T^{z+}(-q)\right) \Gamma}{\Gamma^{2}+\left(\Omega^{\prime}{ }_{0}-\omega\right)^{2}}, \\
& \Gamma=\Gamma_{z+}^{z-}(\omega)=\operatorname{Re} M_{z+}^{z-}, \quad \Omega^{\prime}{ }_{0}=\Omega_{0}+\operatorname{Im} M_{z+}^{z-} .
\end{aligned}
$$

The mass operator $M$ is calculated over the second order in the interaction and zeroth order with respect to the thermodynamic forces. The mass operator determines a position of the resonance line and its width $-\Gamma$.

In accordance with formalism of a chain of equations of motion for Green's functions, the mass operator can be represented as follows: 


$$
\begin{aligned}
M_{-n}^{n}= & \frac{1}{\left(T^{n}(q), T^{-n}(-q)\right)}\left(\left(T^{n}(q),-i q^{k}+\frac{1}{m} T^{-n-k}(-q)\right.\right. \\
& \left.\left.+\dot{T}_{(l)}^{-n}(-q)\right)+G_{2}+G_{1}^{2} G^{-1}\right),
\end{aligned}
$$

where $G_{1}, G_{2}$ are a Green's function type; $m$ is the electron mass.

$$
\begin{aligned}
G_{1}= & \int_{-\infty}^{0} d t e^{\epsilon-i \omega t}\left(T^{n}(q),-i q^{k}+\frac{1}{m} T^{-n-k}(-q, t)\right. \\
& \left.+\dot{T}_{(l)}^{-n}(-q, t)\right), \\
G_{2}= & \int_{-\infty}^{0} d t e^{\epsilon-i \omega t}\left(i q^{-k}+\frac{1}{m} T^{n k}(-q)+\dot{T}_{(l)}^{n}(-q),\right. \\
& \left.-i q^{k}+\frac{1}{m} T^{-n-k}(-q, t)+\dot{T}_{(l)}^{-n}(-q, t)\right) .
\end{aligned}
$$

The real part of the mass operator $\Gamma=\operatorname{Re} M$ plays the role of reciprocal relaxation time of the $T^{n}(q)$ operators. The imaginary part of the mass operator is responsible for a resonance frequency shift.

Finally, for the averaged power absorbed by spin subsection $Q_{s}$ we obtain the following expression

$$
Q_{s}=\beta \sum_{q} \omega^{2}\left|\Phi_{x}^{z-}(q) u^{x}(q)\right|^{2} \frac{\left(T^{z-}(q), T^{z+}(-q)\right) \Gamma}{\Gamma^{2}+\left(\Omega_{0}^{\prime}-\omega\right)^{2}} .
$$

The formula (11) implies that spin conductivity has a resonance at $\Omega_{0}=\omega$.

Keeping only the terms $\sim q^{2}$ in the Born approximation for the resonance line width $\Gamma$, we obtain

$$
\Gamma=\frac{1}{\left(T^{z+}, T^{z-}\right)} \operatorname{Re} G_{2}=q^{2} D+\nu,
$$

where $D$ is the tensor of diffusion:

$$
\begin{aligned}
D= & \frac{m^{-2}}{\left(T^{z+}, T^{z-}\right)} \operatorname{Re} \int_{-\infty}^{0} d t e^{t(\epsilon-i \omega)}\left\{\left(T^{z++}, T^{z--}(t)\right)\right. \\
& \left.+\left(T^{z+-}, T^{z++}(t)\right)\right\}, \\
\nu= & \frac{1}{\left(T^{z+}, T^{z-}\right)} \operatorname{Re} \int_{-\infty}^{0} d t e^{t(\epsilon-i \omega)}\left(\dot{T}_{(l)}^{z+}, \dot{T}_{(l)}^{z-}(t)\right) .
\end{aligned}
$$

Note further that the expression $\nu$ depends on structure of the operators $T^{n}$. Because the spin-independent matrix elements of the electron-impurity interaction are usually much greater than spin scattering matrix elements, $\dot{T}_{l}^{\alpha \beta} \simeq \sum_{i} S_{i}^{\alpha} \dot{p}_{(l)}^{\beta}$. It follows from this that approximately $\nu^{\alpha \beta} \simeq \nu_{p}$, i.e., we have values equal to the relaxation frequency of the electron momentum. The uniform part of a damping of the diffusion tensor has the same value. It follows from formula (13) that the expressions for the diffusion tensor are Green's function of the following type $G_{-\alpha-\eta-\gamma}^{\alpha \eta \gamma} \sim\left(T^{\alpha \eta \gamma}, T^{-\alpha-\eta-\gamma}(t)\right)$ which can be written in a similar way (7) involving the diffusion factors $D_{1}$ and damping factors $\nu_{1}$. Using the explicit form for the correlation functions (5), we get

$$
\frac{\left(T^{z+-}, T^{z-+}\right)}{\left(T^{z+}, T^{z-}\right)} \simeq m^{2} v^{2}
$$

where $v$ is the mean electron velocity. For the line width $\Gamma$ at $\omega_{0}=\omega$, we have

$$
\Gamma \simeq q^{2}\left\{\frac{v^{2} \nu_{p}}{\Omega_{0}^{2}+\nu_{p}^{2}}\right\}+\nu_{p}
$$

In deriving the formula (16), the diffusive part of the diffusion tensor $D$ as a function of damping has been neglected.

Thus the theory holds true when $q^{2} D_{1}<\nu_{1}$. In the case of weak magnetic fields $\Omega_{0}<\nu_{p}$, we have the condition $q l_{\perp}<1$, where $l_{\perp}$ is the mean free path in a plane perpendicular to the magnetic field. For high magnetic fields $\Omega_{0}>\nu_{p}$, we obtain the following criteria $q R<1, q l<1$, where $R$ is cyclotron radius of an electron orbit. The resonance becomes appreciable if $\omega \simeq \Omega_{0}>\Gamma$.

Thus we have shown that the transverse spin current arises when the longitudinal sound wave propagation through the system. In this case, both the average power absorbed by the spin subsystem of the conduction electrons and the spinHall conductivity have a resonant character.

${ }^{1}$ M. I. Dyakonov, Spin Physics in Semiconductors (Springer-Verlag, New York, 2008), pp. 211-244.

${ }^{2}$ V. Sih, R. C. Myers, Y. K. Kato, W. H. Lau, A. C. Gossard, and D. D. Awshalom, "Spatial imaging of the spin Hall effect and current-induced polarization in two-dimensional electron gases," Nat. Phys. 1, 31-35 (2005).

${ }^{3}$ K. Ucida, S. Takahashi, K. Harii, J. Ieda, W. Koshibae, K. Ando, S. Maekawa, and E. Saitoh, "Observation of the spin Seebeck effect," Nature 445, 778-781 (2008).

4" Spin caloritronics" (editorial), Sol. State Comm. 150, 459 (2010).

${ }^{5}$ K. Ucida, H. Adachi, T. An, T. Ota, M. Toda, B. Hillebrands, and S. Maekawa, "Long-range spin Seebeck effect and acoustic spin pumping," Nat. Mat. 10, 737-741 (2011).

${ }^{6}$ N. Mikoshiba, "Ultrasonic spin resonance in metals," Phys. Lett. 12, 289-290 (1964).

${ }^{7}$ E. I. Rashba, "Combined resonance in semiconductors," Sov. Phys. Usp. 7, 823-836 (1965).

${ }^{8}$ A. Overhauser, "Paramagnetic relaxation in metals," Phys. Rev. 89, 689-700 (1953).

${ }^{9}$ Y. Yafet, "g-factors and spin-lattice relaxation of conduction electrons" Solid State Phys. 14, 1-98 (1963).

${ }^{10}$ V. P. Kalashnikov and I. I. Lyapilin, "Nonlinear theory of combined resonance and polarization of nuclei in semiconductors with CdS lattice," Theor. Math. Phys. 18, 194-273 (1974). 
Copyright of Journal of the Acoustical Society of America is the property of American Institute of Physics and its content may not be copied or emailed to multiple sites or posted to a listserv without the copyright holder's express written permission. However, users may print, download, or email articles for individual use. 